\title{
A SOJ A COMO ALIMENTO: VALOR NUTRICIONAL, BENEFÍCIOS PARA A SAÚDE E CULTIVO ORGÂNICO
}

\author{
LUIZ ANTONIO ODENATH PENHA* \\ INÊS CRISTINA DE BATISTA FONSECA** \\ JOSÉ MARCOS MANDARINO*** \\ VERA DE TOLEDO BENASSI ${ }^{\star \star \star *}$
}

\begin{abstract}
Efetuou-se revisão de literatura sobre diversos aspectos ligados à produção e consumo da soja. Abordou-se a importância nutricional da soja, comparando-a com outros alimentos de forma a evidenciar suas vantagens e desvantagens. São mostradas as funções das isoflavonas na fisiologia da planta, como o efeito protetor contra insetos e estimulante da fixação biológica de nitrogênio. São reportados os efeitos da soja no organismo humano, evidenciando seu potencial na redução dos riscos de diversas doenças crônicas e degenerativas e caracterizando-a como alimento funcional. Abordouse também o cultivo da soja em sistema orgânico de modo a situar a inserção da soja nesse contexto. As informações coletadas evidenciaram que o consumo humano da soja e de produtos orgânicos, entre os quais a soja, deve manter crescimento devido maior conhecimento de seus benefícios à saúde.
\end{abstract}

PALAVRAS-CHAVE: Glycine max (L.) Merrill; ISOFLAVONAS; PRODUÇÃO ORGÂNICA; ALIMENTOS FUNCIONAIS.

* Engenheiro Agrônomo, Mestre em Fitotecnia, Secretária da Agricultura e Abastecimento do Paraná, Londrina, PR (e-mail: odenath@pr.gov.br).

* Professora Adjunta, Doutora em Fisiologia Vegetal, Universidade Estadual de Londrina (UEL), Departamento de Agronomia, Londrina, PR (e-mail: inescbr@uel.br).

*** Pesquisador, Mestre em Tecnologia de Alimentos, Embrapa Soja, Londrina, PR (e-mail: jmarcos @cnpso.embrapa.br).

**** Pesquisadora da Embrapa Soja, Doutoranda em Tecnologia de Alimentos na UEL, Londrina, PR (e-mail: benassi@cnpso,embrapa.br). 


\section{INTRODUÇÃO}

A soja, considerada alimento funcional, fornece nutrientes ao organismo e benefícios para a saúde. É rica em proteínas, contém isoflavonas, saponinas, fitatos, inibidores de protease, fitosteróis, peptídeos com baixo peso molecular, oligossacarídeos e ácidos graxos poliinsaturados, que auxiliam na redução de riscos de doenças crônicas e degenerativas. Também constitui boa fonte de minerais como ferro, potássio, magnésio, zinco, cobre fósforo, manganês e vitaminas do complexo B (CARRÃO-PANIZZI e MANDARINO, 1998).

Diversas pesquisas já foram realizadas para melhorar as condições de produtividade, resistência a pragas e doenças, bem como adaptar cultivares de soja a diferentes latitudes e condições edafoclimáticas. O uso da soja e seus derivados para o consumo humano também tem sido objeto de vários estudos (STOLZFUS FEHR e WELKE et al., 2000; DORNBOS JÚNIOR e MULLEN, 1992; GIBSON e MULLEN, 1996; PIPPER e BOOTE, 1999).

Alimentos com contaminantes químicos podem causar danos à saúde a partir de quantidades mínimas (COLBORN, DUMANOSKI e MYERS 2000). Os alimentos produzidos pelo sistema orgânico estão livres de contaminações químicas durante seu cultivo, existindo expectativa dos consumidores de que sejam mais nutritivos do que os produzidos pelo sistema convencional. No entanto, são necessárias mais pesquisas para elucidar essa questão.

Este trabalho de revisão de literatura abrange diversos aspectos nutricionais da soja e seu efeito sobre a saúde humana, enfatizando-se a ação das isoflavonas e a importância do cultivo da soja em sistema orgânico.

\section{A SOJA COMO ALIMENTO FUNCIONAL}

Alimentos funcionais contêm substâncias capazes de modular as respostas metabólicas do indivíduo, resultando em maior proteção e estímulo à saúde. Promovem o bem-estar dos indivíduos, prevenindo o aparecimento precoce de doenças degenerativas e permitindo o aumento da longevidade com qualidade de vida. Portanto, são alimentos que contêm uma ou mais substâncias capazes de atuar no metabolismo ou na fisiologia do ser humano, promovendo benefícios à saúde (PACHECO e SGARBIERI, 2001). As substâncias ou compostos funcionais caracterizam-se por apresentar propriedades que são benéficas ao ser humano como, por exemplo, a ação antioxidante (ANGELIS, 2001). A presença desses componentes nos alimentos é vista como vantagem, que deve ser informada ao consumidor e pode impactar a comercialização do produto. No Brasil, a Agência Nacional de Vigilância Sanitária (ANVISA) definiu normas para rotulagem de produtos com alegação de propriedades funcionais ou de saúde. O alimento ou ingrediente funcional além de suprir funções metabólicas básicas, quando se tratar de nutriente, deve também produzir comprovados efeitos metabólicos, fisiológicos ou benéficos à saúde, devendo ser seguros para o consumo sem supervisão médica (ANVISA, 1999).

A funcionalidade da proteína de soja foi reconhecida em 1999 pelo FDA, órgão de controle de alimentos dos Estados Unidos da América. Foi admitido informar para finalidade de rotulagem nutricional que "dietas com baixo conteúdo de gorduras saturadas e colesterol e que incluam o consumo diário de 25 gramas de proteína de soja podem reduzir os riscos de doenças do coração". A Associação Americana do Coração recomenda o consumo de alimentos com soja para pacientes com elevados níveis de colesterol. Em programas federais de alimentação escolar foi comprovado que a soja pode substituir, sem prejuízo, a proteína animal até o nível de 30\% (MESSINA et al., 2002).

No Brasil, a ANVISA atualizou em janeiro de 2005 a lista de produtos com alegação de benefícios à saúde. São substâncias com propriedades funcionais, de saúde, novos alimentos ou 
ingredientes, substâncias bioativas e probióticos. Para a proteína de soja pode constar a seguinte frase: "o consumo diário de no mínimo 25 g pode ajudar a reduzir o colesterol. Seu consumo deve estar associado com dieta equilibrada e hábitos de vida saudáveis" (ANVISA, 2007).

\section{NUTRIENTES DA SOJA}

O valor nutricional da soja torna-se evidente quando se compara a composição química do leite de vaca e do extrato de soja (Tabela 1 ).

\section{TABELA 1 - COMPOSIÇÃO QUÍMICA DO LEITE DE VACA E DO EXTRATO SOLÚVEL DE SOJA, AMBOS EM PÓ}

\begin{tabular}{lcc}
\hline \multicolumn{1}{c}{ Composição } & Extrato de soja & Leite de vaca \\
\hline Calorias (kcal) & 429,0 & 450,5 \\
Carboidratos (\%) & 28,0 & 35,1 \\
Proteínas (\%) & 41,8 & 28,7 \\
Lipídios (\%) & 20,3 & 21,7 \\
Ca $(\mathrm{mg} / \mathrm{g})$ & 275 & 909 \\
$\mathrm{P}(\mathrm{mg} / \mathrm{g})$ & 674 & 708 \\
$\mathrm{Fe}(\mathrm{mg} / \mathrm{g})$ & 5,0 & 0,5 \\
Retinol (vit. A) $(\mu \mathrm{g} / 100 \mathrm{~g})$ & 4 & 270 \\
Tiamina (vit. B1) $(\mu \mathrm{g} / 100 \mathrm{~g})$ & 300 & 290 \\
Riboflavina (Vit. B2) $(\mu \mathrm{g} / 100 \mathrm{~g})$ & 250 & 1460 \\
Niacina (mg/100 g) & 0,400 & 0,700 \\
Ácido Ascórbico (vit. C) $(\mathrm{mg} / 100 \mathrm{~g})$ & 0,0 & 6,0 \\
\hline
\end{tabular}

Fonte: Franco, 1986.

As proteínas são essenciais na dieta humana e seu valor biológico e nutricional depende da quantidade, digestibilidade, absorção e utilização dos aminoácidos que a compõem (FRIEDMAN e BRANDON, 2001). Na Tabela 2 é mostrada a composição dos aminoácidos essenciais presentes na soja em grão, na farinha, no concentrado e isolado protéico, no extrato de soja, no leite de vaca, e no leite humano em comparação à proteína padrão.

Com porcentagem de proteína de $40 \%$, aproximadamente, a soja apresenta grande interesse para a alimentação como substituta da carne (WILCOX, 1985). Está presente nas dietas vegetarianas, consideradas mais saudáveis, já que os vegetarianos apresentam menor incidência de problemas cardiovasculares (FRIEDMAN e BRANDON, 2001).

O teor de proteínas na soja é definido geneticamente, porém alterações na disponibilidade de nitrogênio para os grãos podem ser induzidas pelo ambiente (HAYATI, EGLI e CRAFTSBRANDNER 1995). Essas alterações na disponibilidade de nitrogênio para os grãos e, conseqüentemente, no teor de proteínas do grão podem estar diretamente relacionadas com a fixação biológica do nitrogênio (PÍPOLO, 2002).

Em condições tropicais, os fatores que mais afetam a fixação biológica de nitrogênio são as altas temperaturas, o estresse hídrico e a acidez do solo. PÍPOLO (2002) observou que a precipitação no período de enchimento dos grãos explica melhor a diferença no teor de proteínas do que as variações na temperatura, devendo esse parâmetro estar ligado ao estresse hídrico. Porém, segundo o mesmo autor, essas variações também influem na produtividade, o que exige maior atenção para se verificar a relação entre o teor de proteínas e os fatores ambientais. Com menor produtividade, o teor de proteínas pode aumentar pela "concentração" da proteína frente ao total de massa seca produzido. 


\section{TABELA 2 - COMPOSIÇÃO RELATIVA DOS AMINOÁCIDOS ESSENCIAIS PRESENTES (g/16 g N TOTAL) NO REQUERIMENTO PADRÃO, NOS LEITES DE VACA E HUMANO E NOS PRODUTOS A BASE DE SOJA}

\begin{tabular}{llllcccc}
\hline Aminoácido & $\begin{array}{c}\text { Padrão } \\
\text { FAO }\end{array}$ & $\begin{array}{c}\text { Leite de } \\
\text { vaca }\end{array}$ & $\begin{array}{c}\text { Leite } \\
\text { humano }\end{array}$ & $\begin{array}{c}\text { Grão de } \\
\text { soja }\end{array}$ & $\begin{array}{c}\text { Farinha de } \\
\text { soja }\end{array}$ & $\begin{array}{c}\text { Extrato de } \\
\text { soja }\end{array}$ & $\begin{array}{c}\text { I.P. de } \\
\text { soja }\end{array}$ \\
\hline Cistina & 4,2 & 1,0 & 2,0 & 1,3 & 1,6 & 1,7 & 1,5 \\
Isoleucina & 4,2 & 7,5 & 5,5 & 4,5 & 4,7 & 5,1 & 4,7 \\
Leucina & 4,8 & 11,0 & 9,1 & 7,8 & 7,9 & 8,3 & 7,8 \\
Lisina & 4,2 & 8,7 & 6,6 & 6,4 & 6,3 & 6,2 & 6,1 \\
Metionina & 2,2 & 3,2 & 2,0 & 1,3 & 1,4 & 1,4 & 1,2 \\
Treonina & 2,8 & 4,7 & 4,5 & 3,9 & 3,9 & 3,8 & 4,2 \\
Triptofano & 1,4 & 1,5 & 1,6 & 1,3 & 1,3 & 1,3 & 1,1 \\
Valina & 4,2 & 7,0 & 6,2 & 4,8 & 5,1 & 4,9 & 4,8 \\
\hline
\end{tabular}

IP = isolado protéico. Fonte: CARRÃO-PANIZZI e MANDARINO, 1998 e MORETTI e GUTIERREZ, 1981.

O estresse hídrico afeta primeiramente a fixação biológica, ocorrendo na seqüência a diminuição da fotossíntese. O resultado é a diminuição da síntese protéica antes de ocorrer a diminuição da produção de massa seca total (SINCLAIR et al., 1987). HUNGRIA e VARGAS (2000) constataram que o estresse hídrico afeta a sobrevivência do Bradyrizobium, bactéria responsável pela fixação biológica de nitrogênio. Também são afetadas a formação e a longevidade dos nódulos, sendo que o estresse mais severo pode levar à paralisação irreversível da fixação de nitrogênio.

O aumento na concentração de proteína, apesar de desejável para a produção de ração animal, nem sempre é favorável. PAEK et al. (1997) e NAKASATHIEN et al. (2000) demonstraram que a elevação no teor de proteínas da soja diminui a qualidade das proteínas. Isso se deve à redução na proporção de aminoácidos sulfurados presente nas proteínas, considerada baixa na soja quando comparada com outras fontes protéicas. A complementação pode ser desejável, mas não é obrigatória para consumo humano, pois dependendo da quantidade de soja ingerida até os aminoácidos sulfurados são supridos.

\section{ISOFLAVONAS DA SOJA}

As isoflavonas são compostos originários do metabolismo secundário, com estrutura básica fenólica. Sua origem química está no ciclo dos ácidos orgânicos (WILDMAN, 2001), sendo formadas durante todo o período de enchimento do grão (desde 35 dias após o florescimento). As formas genistina e malonil genistina aumentam suas concentrações no final do período de enchimento do grão, enquanto as formas daizina e malonil daizina aumentam durante todo o período de enchimento do grão (CARRÃOPANIZZI et al., 1998).

Na Figura 1 estão representadas as 12 formas químicas das isoflavonas presentes na soja, que se diferenciam pelo radical R. As formas agliconas (daidzeína, genisteína, gliciteína) aumentam sua complexidade após se ligarem a uma molécula de glicose (daidzina, genistina, glicitina), depois a um radical acetil (acetil-daidzina, acetil-genistina, acetil-glicitina) e, por fim, a um radical malonil (malonil-daidzina, malonil-genistina, malonil-glicitina).

A absorção e retenção das isoflavonas pelo organismo humano aumentam conforme a solubilidade em água. Desta forma, a genisteína é mais absorvida que a daidzeína que é mais absorvida que a gliciteína. O mecanismo de absorção da genisteína já é conhecido, sendo as isoflavonas glicosiladas inicialmente na mucosa intestinal com a continuidade do processo no fígado. Cerca de $70 \%$ das isoflavonas vão para a bile quatro horas após sua ingestão e $25 \%$ da excreção ocorre pela urina (HENDRICH e MURPHY, 2001). 


\section{FIGURA 1 - ISOFLAVONAS PRESENTES NA SOJA}

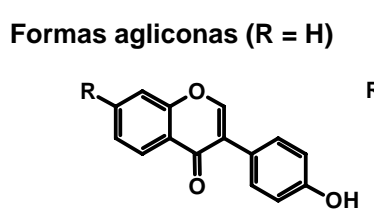

$\mathrm{R}=\mathrm{H}$
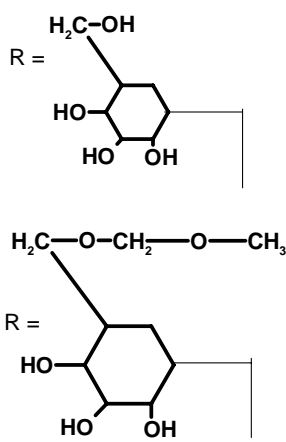

6-O-acetil

Daidzina

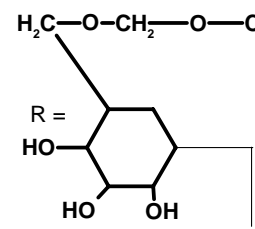

Daidzina

Daizeína<smiles>[R]c1cc(O)c2c(=O)c(-c3ccc(O)cc3)coc2c1</smiles>

Genisteína

Genistina

6-O-acetil

Genistina

6-0-malonil

Genistina<smiles></smiles>

Glicitina
Gliciteína

6-O-acetil

Gliciteína

Fonte: Adaptado de FRIEDMAN e BRANDON, 2001.

\section{AÇÃO DAS ISOFLAVONAS NO HOMEM}

As isoflavonas podem funcionar como repositores hormonais naturais. Sua ação baseia-se na capacidade de ligação com os receptores de estrógeno. Atuam na translocação para o núcleo da célula e induzem a transcrição do gene específico, que pode incluir a divisão celular. Uma das formas de ação das isoflavonas, particularmente da genisteína, é o bloqueio de fosforilações específicas como a do fator NFkB que participa em processos inflamatórios e na osteoporose. $\mathrm{O}$ consumo de isoflavonas provoca efeito antiangiogênico, inibição da DNA topoisomerase e seus efeitos antioxidantes em lipídios. Agem sobre lipoproteínas e DNA, bem como no transporte de glicose e em vários sistemas de transportes de íons (BARNES, KIM e XU, 2002).

As isoflavonas podem atuar tanto como agonistas quanto antagonistas do estrógeno, ou como moduladores dos receptores seletivos de estrógeno. Seu efeito varia dependendo do órgão do corpo humano, do tipo de célula, da concentração de isoflavonas, da condição hormonal, idade, etc. (BADGER et al., 2002).

Aplicação bastante conhecida das isoflavonas diz respeito à pós-menopausa. A terapia de reposição hormonal (TRH) é freqüentemente prescrita para mulheres em fase de climatério. Esse tratamento previne a perda da densidade óssea, que pode causar osteoporose. Porém, o uso de hormônios sintéticos aumenta o risco de doenças cardíacas, assim como o risco de câncer endometrial (BARNES, KIM e XU, 2002). 
As isoflavonas apresentam atividade estrogênica fraca (CHENG et al., 1953; SONG, HENDRICH e MURPHY 1999), significando menor atividade hormonal no corpo humano em relação aos hormônios sintéticos. Esse fator tem sido considerado nos recomendações de cápsulas a base de isoflavonas, ou da própria soja e seus derivados para evitar problemas de superdosagem e possíveis efeitos deletérios da reposição hormonal química.

O consumo de soja (cerca de $25 \mathrm{~g} /$ dia de proteína) não diminuiu o número de ondas de calor nas mulheres em fase de climatério, mas reduz sua intensidade (HAN et al.,2001; CLARKSON, 2002; SCHEIBER, 2001). Em quantidades maiores (40-60 g/dia de proteína), o número de ondas de calor diminui em $40 \%$ podendo decrescer mais ainda com a ingestão de extratos concentrados de isoflavonas (106 mg de isoflavonas/dia) (HAN et al.,2001; CLARKSON, 2002; SCHEIBER, 2001).

Segundo diversos autores (BARNES, KIM e XU 2002; TSUKAMOTO et al., 2001; MARANHÃO, 2001; HAN et al., 2001), o consumo de isoflavonas deve ser de aproximadamente $50 \mathrm{mg}$ por dia para que ocorram efeitos benéficos. Vale lembrar que a concentração de isoflavonas nas diferentes cultivares de soja varia muito devido a fatores genéticos da planta e condições climáticas durante o cultivo. Outros estudos relacionam os efeitos da soja com a prevenção de câncer. Segundo MESSINA et al. (1994), as isoflavonas apresentaram efeito comprovado de proteção contra a carcinogênese em testes com animais (65\% de resultados positivos). A ação protetora das isoflavonas em câncer de próstata, mama e outras formas de câncer hormônio dependentes em humanos já foi constatada (BRADLOW e SEPKOVIC, 2002; BENNINK, 2001; MESSINA et al., 1994; KENNEDY, 1995; CARAGAY, 1992). Esse efeito protetor ocorre por meio da regulação dos esteróides sexuais específicos e de fatores de crescimento dos tumores, conforme identificaram LAMARTINIERE et al. (2002) estudando a ingestão de genisteína.

$\mathrm{O}$ isolado protéico de soja reduziu a incidência de tumores e prolongou o tempo para o aparecimento de novos tumores em modelos experimentais com ratos. Experimentos com soja fermentada também mostraram diminuição na incidência, número e tamanho de tumores, segundo BENNINK (2001).

JENKINS et al. (2003) estudaram o uso de isoflavonas por indivíduos com câncer de próstata, tendo obtido resultados positivos em termos de prevenção e de tratamento. APUZZIO (2003) detectou efeito positivo do uso de isoflavonas como forma de prevenção do câncer de mama em mulheres na fase de pré-menopausa. A ingestão de isoflavonas desde a infância produz maior efeito na redução dos riscos de câncer, embora a dieta com soja a partir da fase adulta também possa trazer benefícios (WU et al., 2002).

A genisteína constitui fator importante na prevenção de doenças inflamatórias e autoimunes, devido ação inibidora da fosforilação da tirosina e supressão da síntese induzida de óxido nítrico (SADOWSKA-KROWICKA, 1998).

Efeitos sobre o sistema circulatório também foram estudados por ARLISS e BIERMANN (2002), que constataram diminuição no nível de triglicerídeos em humanos e a inibição da aterosclerose em animais submetidos à dieta com soja.

CLARKSON (2002) e NESTEL (2003) relataram efeitos positivos da soja para a prevenção da aterosclerose. Esse último autor estudou também os efeitos relacionados à melhora do sistema cardiovascular mediante consumo de isoflavonas, assim como FUHRMAN e AVIRAM (2001), HASLER (2002) e JAYAGOPAL (2002). A redução nos níveis de colesterol pelo uso de isoflavonas foi identificada por JENKINS et al. (2003), HASLER (2002), MADDOX et al. (2002), CLARKSON (2002) e MARANHÃO (2001). Os mesmos efeitos foram observados por FRIEDMAN e BRANDON (2001) quando utilizaram, em seus experimentos, a proteína de soja.

A longevidade em humanos, associada ao consumo de isoflavonas, foi estudada por NAGATA, TAKATSUKA e SHIMIZU (2002). A pesquisa durou 7 anos e envolveu 13.355 homens e 15.724 mulheres (japoneses). Mesmo ajustando fatores associados à dieta, o resultado mostrou que o consumo de soja pode ter efeitos benéficos moderados perante a mortalidade total. 
GENOVESE e LAJOLO (2002) estudaram os teores de isoflavonas em alimentos brasileiros, preocupados com a possibilidade do consumo excessivo alterar o crescimento de crianças em formação pelo efeito hormonal das isoflavonas. Verificaram que a quantidade de isoflavonas presente nos alimentos a base de soja varia de $2 \mathrm{a} 100 \mathrm{mg} / 100 \mathrm{~g}$ (base seca) e consumo entre as crianças brasileiras de 1,6 a $6,6 \mathrm{mg} / \mathrm{kg}$ de peso corporal, não identificando nenhum fator de risco.

Muitos efeitos benéficos da soja para a saúde humana são atribuídos, tanto às proteínas quanto às isoflavonas. Há evidencias de forte efeito sinérgico entre proteínas e isoflafonas, ou outros compostos desconhecidos (BARNES, KIM e XU, 2002). Seu efeito notório sobre a população oriental ocorre a partir do consumo regular de pequenas quantidades, mais como complemento alimentar do que como base da alimentação.

\section{AÇÃO DE OUTROS COMPONENTES DA SOJA}

Aproximadamente $6 \%$ das proteínas de soja são constituídas pelos inibidores de tripsina e quimotripsina, principalmente o inibidor de tripsina de Kunitz (ITK) e o inibidor de tripsina e quimotripsina de Bowman-Birk (ITQBB) (BRANDON e FRIEDMAN, 2002). Esses inibidores de proteases estão presentes em diversos alimentos, como cereais, batatas e tomates. A ingestão de alimentos em que os mesmos encontram-se ativos pode causar redução no ganho de peso de animais. A inativação ocorre por meio de processamentos térmicos, como cozimento ou torra, restando entre 5 a 20\% de ITK e ITQBB nos alimentos de soja comercialmente processados (FRIEDMAN e BRANDON, 2001).

Os inibidores de protease apresentam efeitos anticarcinogênicos, provavelmente, devido à sua interação com a serina celular protease (BRANDON e FRIEDMAN, 2002). O modo de ação envolveria o bloqueio da criação de formas de oxigênio ativo por neutrófilos estimulados, inibindo assim o crescimento do tumor. Outro mecanismo proposto seria a indisponibilidade de aminoácidos, resultante do bloqueio ou diminuição da "digestão" de proteínas, impedindo assim o rápido crescimento dos tumores (FRIEDMAN e BRANDON, 2001).

Segundo FRIEDMAN e BRANDON (2001) o ITQBB pode reduzir os riscos de câncer de mama, provavelmente devido à inibição da produção de radicais livres. Há também evidências quanto à redução nos riscos de câncer na cabeça, pescoço, fígado, boca, ovário e cervical. Além disso, os benefícios da dieta a base de soja incluem diminuição na progressão de doenças renais em pacientes renais crônicos.

As fibras insolúveis da soja não são digeridas no trato gastrointestinal humano e atuam normalizando a mobilidade intestinal, o que previne diverticulite e constipação. As fibras solúveis são efetivas no controle do diabetes tipo II (pacientes não insulino-dependentes) e na redução dos níveis sangüíneos de LDL-colesterol (CHANG, 2001).

\section{PRODUÇÃO ORGÂNICA DE ALIMENTOS}

Os produtos químicos utilizados na agricultura convencional causam distúrbios fisiológicos que, algumas vezes, não podem ser identificados diretamente. Contaminações da ordem de parte por trilhão (ppt) podem causar efeitos adversos no organismo com danos sutis como, por exemplo, alterações hormonais que não podem ser prontamente identificadas (COLBORN, DUMANOSKI e MYERS 2000). Esses produtos químicos podem promover alterações metabólicas nos microorganismos associados aos vegetais, provocando o desequilíbrio de ambos. Isso resulta em fragilidade no vegetal frente à disputa com pragas e microorganismos e dá lugar à manifestação de doenças. A contaminação química também prejudica a resistência dos vegetais às condições de 
estresse ambiental e fisiológico (TOKESHI, 2000). O acesso a essas informações tem estimulado consumidores a buscarem produtos orgânicos. Como conseqüência, está ocorrendo o aumento da produção de soja orgânica para responder à demanda de consumidores por produtos considerados mais saudáveis. Esse conceito inclui composição equilibrada dos aminoácidos presentes nas proteínas, e baixos níveis ou ausência de contaminação por metais pesados ou agrotóxicos (SMITH et al., 1993; KUMPULAINEN, 2001).

Segundo o Instituto Biodinâmico (IBD), instituição certificadora brasileira que verifica a conformidade dos produtos orgânicos com as normas nacionais e internacionais, as característica do cultivo orgânico são: 1) proteção da fertilidade dos solos a longo prazo, estimulando sua atividade biológica; 2) intervenção mecanizada cautelosa; 3) fornecimento de nutrientes ao solo de forma natural, não utilizando fertilizantes obtidos por processos químicos; 4) auto-suficiência em nitrogênio pelo uso de leguminosas, inoculação com bactérias fixadoras de nitrogênio e reciclagem de materiais orgânicos provenientes de resíduos vegetais e esterco de animais; 5) controle de doenças, pragas e plantas daninhas pela rotação de culturas, inimigos naturais, diversidade genética, variedades resistentes, adubação orgânica, intervenções biológicas, extratos elaborados com plantas e outros componentes naturais; 6 ) bem-estar das espécies exploradas na criação animal por meio da nutrição, tratamento sanitário e condições de vida que respeitem suas características; 7) atenção especial ao impacto do sistema produtivo sobre o ambiente, protegendo a flora e a fauna existentes; 8) condições de trabalho que representem oportunidade de desenvolvimento humano aos envolvidos; 9) processamento limpo e controlado; e 10) extrativismo sustentável (INSTITUTO BIODINÂMICO, 2003)

WEIBEL et al. (2000) encontraram aumento de 19\% no conteúdo de fenóis em produtos orgânicos comparados com produtos convencionais, a maioria flavonóides (a cujo grupo pertencem as isoflavonas). Outras características, como teor de fósforo, teste de degradação e teste de preferência alimentar com ratos também foram consideradas melhores em produtos orgânicos.

Vários autores verificaram diferenças entre produtos de origem orgânica e convencional, quanto ao conteúdo de minerais e características sensoriais (SMITH, 1993; DEELL e PRANGE, 1993; WARMAN e HAVARD, 1997; FJELKNER-MODIG et al., 2000; KUMPULAINEN, 2001).

Os produtos orgânicos são também procurados pelos consumidores devido ao apelo ambiental de proteção à natureza. HUSBERGEN e KALK (2001) identificaram menor consumo de energia fóssil por área no sistema de produção orgânico do que no convencional. REGANOLD et al. (2001) também evidenciaram menor impacto ambiental na produção pelo sistema orgânico. PIMENTEL et al. (2005) acrescentaram a essas informações maior retenção de água no solo, aumento da biodiversidade e maior sustentabilidade no sistema orgânico.

Foram observadas melhorias no sistema de produção orgânico relativas à estrutura e atividade microbiana do solo, como redução do risco de erosão e aumento na quantidade de predadores/inimigos naturais de pragas. Há indícios, também, de que o sistema orgânico aumente a resistência ao estresse e distúrbios externos (LEIBIG e DORAN, 1999; FLIESSBACH et al., 2000; REGANOLD et al., 2001; CARPENTER, KENNEDY e REGANOLD 2000; CLARK et al., 1998.).

Dados econômicos sobre a produção orgânica no Brasil são raros e dispersos, sendo o tratalho de ORMOND et al. (2002) um dos mais completos. A produção nacional orgânica foi estimada em US\$ 150 milhões em 1999, sendo US\$ 20 milhões para o mercado interno. Esse valor pode chegar a US\$ 300 milhões, quando acrescido de frutas e palmito, além da margem dos distribuidores. Consta desse estudo o levantamento de 7.063 produtores brasileiros certificados, com 270 mil hectares, sendo 116 mil para pastagem. A soja tem a maior quantidade (593) de produtores certificados $(8,4 \%)$, seguida pelas hortaliças com 549 produtores $(7,8 \%)$, representando áreas de 12,5 mil e 3 mil hectares, respectivamente. O mesmo documento apresenta dados conflitantes sobre produtores não-certificados, citando áreas com hortaliças próximas a 9,5 mil hectares apenas nos estados da região Sul. 
Estimativas para 2006 indicam produção nacional certificada em torno de US\$250 milhões, o que é pouco considerando que o mercado mundial alcança US $\$ 30$ bilhões (CZAPSKI, 2007). Para o mesmo autor, o comércio informal ou com certificação não-contratada somaria cerca de US\$30 milhões anuais ao volume comercializado no Brasil.

Apesar de alguns resultados contrários e da preocupação quanto ao consumo excessivo de isoflavonas em longo prazo, o número de trabalhos comprovando seus efeitos benéficos é imensamente superior. Por tratar-se de substância naturalmente presente na soja, consumida pelo homem há milhares de anos como alimento, existe grande experiência acumulada quanto à segurança de sua ingestão (muito superior à evidenciada por ensaios biológicos ou estudos epidemiológicos). Seu consumo no oriente é feito em pequenas quantidades, como complemento alimentar, e não como substituto do feijão consumido no Brasil, conforme mostrado pelos dados de consumo per capita da FAO (2005).

\title{
8 CONSIDERAÇÕES FINAIS
}

Diante dos benefícios da produção orgânica resta a missão, já encampada por vários produtores, de tornar cada vez mais acessível o alimento orgânico em termos de preço e oferta que ainda é insuficiente para atender a toda a demanda existente.

Apesar da necessidade de se combater a fome, existe também a necessidade de se promover a saúde. De fome padecem muitos pobres, mas de falta de saúde padecem esses e muitos mais, sendo que atualmente aumentam cada vez mais os problemas devidos à obesidade e ao consumo excessivo de alimentos. A soja, especialmente aquela produzida pelo sistema orgânico, pode ajudar na resolução de ambos os problemas. Trata-se de alimento nutricionalmente rico, servindo para saciar a fome e nutrir o organismo, colaborando para a manutenção da saúde da população em geral.

\begin{abstract}
SOYBEAN AS FOOD: NUTRITIONAL VALUE, BENEFITS FOR HEALTH AND ORGANIC CULTIVATION

A literature review was accomplished about several aspects related to soyben production and consumption. Soybean nutritional value and its importance as food are presented comparing to other foodstuffs in a way to evidence its advantages and disadvantages. Isoflavones effects on plant physiology are mentioned, as well as their protective effect against insects and stimulatory effect of nitrogen biological fixation. The effects of soybeans on human body was also described, evidencing its potential to reduce risks of several chronic and degenerative diseases, which characterizes soybeans as functional food. Soybean organic production was mentioned in a way to situate the insertion of soybeans in this context. The collected information evidenced that human consumption of soybeans and organic products, among them soybeans, will grow continuously influenced by knowledge of their health benefits.
\end{abstract}

KEY-WORDS: Glycine max (L.) Merrill; ISOFLAVONES; ORGANIC PRODUCTION; FUNCTIONAL FOODS.

\section{REFERÊNCIAS}

1 ANVISA. Resolução 18, de 30 de abril de 1999. Diretrizes básicas para análise e comprovação de propriedades funcionais e ou de saúde alegadas em rotulagem de alimentos. Diário Oficial [da] Republica Federativa do Brasil, Brasília, seção 1, de 03 de dezembro de 1999.

2 ANVISA. Alimentos com alegações de propriedades funcionais e ou de saúde, novos alimentos/ingredientes, substâncias bioativas e probióticos. Disponível em <http://www.anvisa.gov.br/alimentos/comissoes/ tecno_lista_alega.htm>. Acesso em: 30 mar. 2007

3 APUZZIO, D.M. Isoflavones may help prevent premenopausal ER-positive breast cancer. Physician Assistant, v. 27, n. 1, p. 35-38, 2003.

4 ARLISS, R.M.; BIERMANN, C.A. Do soy isoflavones lower cholesterol, inhibit arteriosclerosis, and play a role in cancer 
prevention? Holistic Nursing Practice, v. 17, n.1, p. 40-48, 2002.

5 BADGER, T.M.; RONIS, M.J.J.; HAKKAK, R.; ROWLANDS, J.C.; KOROURIAN, S. The health consequences of early soy consumption. Journal of Nutrition, v. 132, n. 3, p. 559S-565S, 2002.

6 BARNES, S.; KIM, H.; XU, J. Soy in the prevention and treatment of chronic diseases. In: CONGRESSO BRASILEIRO DE SOJA, 2., 2002, Foz do Iguaçu. Anais... Londrina: Embrapa, 2002. p.295-308

7 BENNINK, M.R. Soybean in the prevention and treatment of cancer. In: SIMPÓSIO BRASILEIRO SOBRE OS BENEFÍCIOS DA SOJA PARA A SAÚDE HUMANA, 1., 2001, Londrina. Anais... Londrina: Embrapa Soja, 2001. p.2427.

8 BRADLOW, H.L.; SEPKOVIC, D.W. Diet and breast cancer. Annals of the New York Academy of Sciences, v. 963, p. 247-267, 2002.

9 CARAGAY, A.B. Cancer-preventive foods and ingredients. Food Technology, v.46, p. 65-68, 1992.

10 CARPENTER B.L.; KENNEDY, AC.; REGANOLD, J.P. Organic and biodynamic management: effects on soil biology. American Journal of Soil Science Society, v. 64, p.1651-1659, 2000.

11 CARRÃO-PANIZZI, M.C.; KITAMURA, K.; BELÉIA, A.D.P.; OLIVEIRA, M.C.N. Influence of growth locations on isoflavone contents in brazilian soybean cultivars. Breeding Science, v. 48, p. 409-413, 1998.

12 CARRÃO-PANIZZI, M.C.; MANDARINO, J.M.G. Soja: potencial de uso na dieta brasileira. Londrina: Embrapa-CNPSo, 1998. (Embrapa-CNPSo. Documento, 113)

13 CHANG, Y.K. Alimentos funcionais e aplicação tecnológica: padaria da saúde e centro de pesquisas em tecnologia de extrusão. In: SIMPÓSIO BRASILEIRO SOBRE OS BENEFÍCIOS DA SOJA PARAA SAÚDE HUMANA, 1., 2001, Londrina. Anais... Londrina: Embrapa Soja, 2001. p. 41-45.

14 CHENG, E.; STORY, C.D.; YODER, L.; HALE, W.H.; BURROUGHS, W. Estrogenic activity of isoflavone derivatives extracted and prepared from soybean oil meal. Science, v. 118, p. 164-165, 1953.

15 CLARK, M.S.; HORWATH, W.R.; CENAN, C.; SHOW, K.M. Changes in soil chemical properties resulting from organic and low-input farming practices. Agronomy Journal, v. 90, p. 662-671, 1998.

CLARKSON, T.B. Soy, soy phytoestrogens and cardiovascular disease. Journal of Nutrition, v. 132, n. 3, p. 566S-569S, 2002.

17 COLBORN, T.; DUMANOSKI, D.; MYERS, J.P. O futuro roubado. Porto Alegre: L\&PM Editores, 2000. 354 p.

18 CZAPSKI, S. Mercado de orgânicos aguarda regulamentação. Disponível em: <http://www.organicosbrasil.org/ noticias_ver.php?id=73\&id_arvore=5>. Acesso em: 02 abr. 2007.

19 DEELL, J.R.; PRANGE, R.K. Post harvest physiological disorders, diseases and mineral concentrations of organically and conventionally grown McIntosh and Cortland apples. Canadian Journal of Plant Science, v. 73, p. 223-230, 1993.

20 DORNBOS JUNIOR, D.L.; MULLEN, R.E. Soybean seed protein and oil contents and fatty acid composition adjustment by drought and temperature. Journal of American Oil Society, v. 69, p. 228-231, 1992.

21 FAO. Food And Agriculture Organization. FAOSTAT DATABASE QUERY. Disponível em <http://faostat.fao.org/faostat/ form? collection=FS.CropsAndProducts\&Domain=FS\&servlet=1\&hasbulk=\&version=ext\&language=EN > . Acesso em: 20 dez. 2005

22 FJELKNER-MODIG, S.; BENGTSSON, H.; STEGMARK, R.; NYSTRÕM, S. The influence of organic and integrated production on nutritional, sensory and agricultural aspects of vegetable raw materials for food production. Soil and Plant Science, v. 50, p. 102-113, 2000.

23 FLIESSBACH, A; MADER, P; NIGGLI, U. Mineralization and microbial assimilation of 14C-labeled straw in soils of organic and conventional agricultural systems. Soil Biology and Biochemistry, v. 32, p. 1131-1130, 2000.

24 FRANCO, G. Tabela de composição de alimentos. Rio de Janeiro: Athenen, 1986.

25 FRIEDMAN, M.; BRANDON, D.L. Nutritional and health benefits of soy proteins. Journal of Agriculture and Food Chemistry, v. 49, n.3, p. 1069-1086, 2001.

26 FUHRMAN, B.; AVIRAM, M. Flavonoids protect from oxidation and attenuate atherosclerosis. Current Opinion in Lipidology, v. 12, n. 1, p. 41-48, 2001.

27 GENOVESE, M.I.; LAJOLO, F.M. Isoflavones in soy-based foods consumed in Brazil: levels, distribution, and estimated intake. Journal of Agriculture and Food Chemistry, v. 20, p. 5987-5993, 2002. 
28 GIBSON, L.R.; MULLEN, R.E. Soybean composition under high day and night growth temperatures. Journal of American Oil Society, v. 73, p. 733-737, 1996.

29 HAN, K.K.; KATI, L.M.; HAIDAR, M.A.; GIRÃO, M.J.B.C.; BARACAT, E.C.; YIM, D.K.; CARRÃO-PANIZZI, M.C. Efeito de isoflavonas sobre os sintomas da síndrome de climatério. In: SIMPÓSIO BRASILEIRO SOBRE OS BENEFÍCIOS DA SOJA PARAA SAÚDE HUMANA, 1., 2001, Londrina. Anais... Londrina: Embrapa Soja, 2001. p. 28-32. (Embrapa Soja. Circular, 169)

30 HASLER, C.M. The cardiovascular effects of soy products. Journal of Cardiovascular Nursing, v. 16, n. 4, p. 50-63, 2002.

31 HAYATI, R.; EGLI, D.B.; CRAFTS-BRANDNER, S.J. Carbon and nitrogen supply during seed filling and leaf senescence in soybean. Crop Science, v.35, p.1063-1069, 1995.

32 HENDRICH, S.; MURPHY, P.A. Isoflavones: source and metabolism. In: HANDBOOK of nutraceuticals and functional foods. Boca Raton: CRC Press, 2001. p. 55-72.

33 HUNGRIA, M.; VARGAS, M.A.T. Environmental factors affecting N2 fixation in grain legumes in the tropics, with an emphasis on Brazil. Field Crops Research, v. 65, p.151-164, 2000.

34 HUSBERGEN, K.J.; KALK, W.D. Energy balances in different agricultural systems - can they be improved. In: SYMPOSIUM OF THE INTERNATIONAL FERTILIZERS SOCIETY, Lisboa, 2001. Proceedings... York: International Fertilizers Society, 2001. p. 20-36.

35 INSTITUTO BIODINÂMICO. Diretrizes Orgânico Instituto Biodinâmico Disponível em <http://www.ibd.com.br/arquivos/ agriculturaorganica /agriculturaorganica.htm.>. Acesso em: 10 abr. 2003.

36 JAYAGOPAL, V.; ALBERTAZZI, P.; KILPATRICK, E.S.; HOWARTH, E.M.; JENNINGS, P.E.; HEPBURN, D.A.; ATKIN, S.L. Beneficial effects of soy phytoestrogen intake in postmenopausal women with type 2 diabetes. Diabetes Care, v. 25 , n.10, p.1709-1714, 2002

37 JENKINS, D.J.A.; KENDALL, C.W.C.; D'COSTA, M.A.; JACKSON, C.; VIDGEN, E.; SINGER, W.; SILVERMAN, J.A.; KOUMBRIDIS, G.; HONEY, J.; VENKET, R.A.; FLESHNER, N.; KLOTZ, L. Soy consumption and phytoestrogens: effect on serum prostate specific antigen when blood lipids and oxidized low-density lipoprotein are reduced in hyperlipidemic men. Journal of Urology, v. 169, n. 2, p. 507-511, 2003.

38 KENNEDY, A.R. The evidence for soybean products as cancer preventive agents. American Institute of Nutrition, v. 125, Supplement, p. 733s-743s, 1995

39 KUMPULAINEN, J. Nutritional and toxicological quality comparisons between organic and conventionally grown foodstuffs. In: SYMPOSIUM OF THE INTERNATIONAL FERTILIZERS SOCIETY, Lisboa, 2001. Proceedings... York: International Fertilizers Society, 2001. p.1-20.

40 LAMARTINIERE, C.A.; COTRONEO, M.S.; FRITZ W.A.; WANG, J.; MENTOR-MARCEL, R.; ELGAVISH, A. Genistein chemoprevention: timing and mechanisms of action in murine mammary and prostate. Journal of Nutrition, v. 132, n. 3, p. 552S-558S, 2002.

41 LEIBIG, M.A; DORAN, J.W. Impact of organic production practices on soil quality indicators. Journal of Environmental Quality, v. 28, p. 1601-1609, 1999.

42 LOH, J.T. et al. Population density-dependent regulation of the Bradyrhizobium japonicum nodulation genes. Molecular Microbiology, v. 42, n. 1, p. 37-46, 2001.

43 MADDOX, D.A.; ALAVI, F.K; SILBERNICK, E.M.; ZAWADA Jr, E.W. Protective effects of a soy diet in preventing obesitylinked renal disease. Kidney International, v.61, n.1, p. 96-104, 2002.

44 MARANHÃO, M.F.C. Benefícios da soja para o coração e a saúde. In: SIMPÓSIO BRASILEIRO SOBRE OS BENEFÍCIOS DA SOJA PARA A SAÚDE HUMANA, 1., 2001, Londrina. Anais... Londrina: Embrapa Soja, 2001. p. 21-23. (Embrapa Soja. Circular, 169)

45 MESSINA, M.J.; PERSKY,V.; SETCHELL, K.D.; BARNES, S. Soy intake and cancer risk: a review of the in vitro and in vivo data. Nutrition and Cancer, v. 21, p. 113-121, 1994.

46 MORETTI, R.H.; GUTIERREZ, R.H. Produção de soja em escala semi-industrial. In: MIYASAKA, S.; MEDINA, J.C. (eds). A soja no Brasil. Campinas, ITAL, 1981. p. 979-986.

47 NAGATA, C.; TAKATSUKA, N.; SHIMIZU, H. Soy and fish oil intake and mortality in a japanese community. American Journal of Epidemiology, v.156, n.9, p.824-831, 2002.

48 NAKASATHIEN, S.; ISRAEL, D.W.; WILSON, R.F.; KWANYUEN, P. Regulation of seed protein concentration in soybean by supra-optimal nitrogen supply. Crop Science, v. 40, p. 1277-1284, 2000. 
49 NESTEL, P. Isoflavones: their effects on cardiovascular risk and functions. Current Opinions on Lipidology, v. 14, n. 1, p. 3-8, 2003

50 ORMOND, J.G.P.; PAULA, S.R.L.; FAVERET FILHO, P.; ROCHA, L.T.M. Agricultura orgânica: quando o passado é futuro. BNDES setorial, Rio de Janeiro, n. 15, p. 3-34, mar. 2002.

51 PACHECO, M.T.B.; SGARBIERI, V.C. Alimentos Funcionais: conceituação e importância na saúde humana. In: SIMPÓSIO BRASILEIRO SOBRE OS BENEFÍCIOS DA SOJA PARAA SAÚDE HUMANA, 1., 2001, Londrina, 2001. Anais... Londrina: Embrapa Soja, 2001. p. 37-40

52 PAEK, N.C.; INSANDE, J.; SHOEMAKER, R.C.; SHIBLE, R. Nutritional control of soybean seed storage protein. Crop Science, v. 37, p. 498-503, 1997.

53 PIMENTEL, D.; HEPPERLY, P.; HANSON, J.; DOUDS, D.; SEIDEL, R. Environmental, energetic and economic comparisons of organic and conventional farming systems. Bioscience, v. 55, p.573-582, 2005.

54 PÍPOLO, A. E. Influência da temperatura sobre as concentrações de proteína e óleo em sementes de soja (Glycine $\max ($ L.) Merrill). 2002. 128 f. Tese (Doutorado em Agronomia) - Escola Superior de Agricultura "Luiz de Queiroz" Universidade de São Paulo, Piracicaba.

55 PIPPER, E.L.; BOOTE, K.J.Temperature and cultivar effects on soybean seed oil and protein concentration. Journal of American Oil Society, v. 76, p. 1233-1241, 1999.

56 REGANOLD, J.P. et al. Sustainability of three apple production systems. Nature, v. 410, p. 926-929, 2001.

57 SADOWSKA-KROWICKA, H. et al., Genistein and gus inflammation: role of nitric oxid. Proceedings of the Society for Experimental Biology \& Medicine, Bisset, v. 217, p. 315-357, 1998.

58 SCHEIBER, M.D. et al. Dietary inclusion of whole soy foods results in significant reductions in clinical risk factors for osteoporosis and cardiovascular disease in normal postmenopausal women. Menopause, v. 8, n. 5, p. 384-392, 2001.

59 SINCLAIR, T.R. Relative sensitivity of nitrogen and biomass accumulation do drought in field-grown soybean. Agronomy Journal, v. 79, p. 986-991, 1987.

60 SMITH, B.L. Comparison of element levels in organic foods and commercial foods. In: INTERNATIONAL SYMPOSIUM ON TRACE ELEMENTS IN MAN AND ANIMALS, 8., Gersdorf, 1993. Proceedings..., Gersdorf: Media Touristik, 1993. p. $255-256$.

61 SONG, T.T.; HENDRICH, S.; MURPHY, P.A. Estrogenic activity of glycitein, a soy isoflavon. Journal of Agriculture and Food Chemistry, v. 47, p. 1607-1610, 1999.

62 STOLZFUS, D.L.; FEHR, W.R.; WELKE, G.A. Relationship of elevated palmitate to soybean seed traits. Crop Science, v. 40 , p. $52-54,2000$.

63 TOKESHI, H. Doenças e pragas agrícolas geradas e multiplicadas pelos agrotóxicos. Fitopatologia Brasileira, v. 25, p. 264-271, 2000.

64 TSUKAMOTO, C.; KUDOU, S.; KIKUCHI, A.; CARRÃO-PANIZZI, M.C.; ONO, T.; KITAMURA, K.; OKUBO, K. Isoflavones in soybean products: composition, concentration and physiological effects. In: SIMPÓSIO BRASILEIRO SOBRE OS BENEFÍCIOS DA SOJA PARA A SAÚDE HUMANA, 1., 2001, Londrina, 2001. Anais... Londrina: Embrapa Soja, 2001. p. 9-14. (Embrapa Soja. Circular, 169)

65 WARMAN, P.R; HAVARD, K.A. Yield, vitamin and mineral contents of organically and conventionally grown potatoes and sweet corn. Agriculture, Ecosystems and Environment, v. 68, p. 207-216, 1997.

66 WEIBEL, F.P. et al. Are organically grown apple tastier and healthier? A comparative field study using conventional and alternative methods to measure fruit quality. Acta Horticulturae, v. 517, p. 417-426, 2000.

67 WILCOX, J.R. Breeding soybeans for improved oil quantity and quality. In: WORLD SOYBEAN RESEARCH CONFERENCE, 3., Boulder, 1985. Proceedings... Boulder: Westview Press, 1985. p. 380-386.

68 WILDMAN, R.E.C. (Ed.). Handbook of nutraceuticals and functional foods. Boca Raton: CRC Press, 2001.542 p.

69 WU, A. et al. Adolescent and adult soy intake and risk of breast cancer in asian-americans. Carcinogenesis, v. 23, n. 9, p. 1491-1496, 2002 